\title{
Work area monitoring in dynamic environments using multiple auto-aligning 3-D sensors
}

\author{
Y. Wang, D. Ewert, T. Meisen, D. Schilberg, and S. Jeschke \\ Institute Cluster IMA/ZLW \& IfU Institute, RWTH Aachen University, Germany \\ Correspondence to: Y. Wang (ying.wang@ima-zlw-ifu.rwth-aachen.de)
}

Received: 29 November 2013 - Revised: 4 March 2014 - Accepted: 6 March 2014 - Published: 3 June 2014

\begin{abstract}
Compared to current industry standards future production systems will be more flexible and robust and will adapt to unforeseen states and events. Industrial robots will interact with each other as well as with human coworkers. To be able to act in such a dynamic environment, each acting entity ideally needs complete knowledge of its surroundings, concerning working materials as well as other working entities. Therefore new monitoring methods providing complete coverage for complex and changing working areas are needed. While single 3-D sensors already provide detailed information within their field of view, complete coverage of a complete work area can only be achieved by relying on a multitude of these sensors.

However, to provide useful information all data of each sensor must be aligned to each other and fused into an overall world picture. To be able to align the data correctly, the position and orientation of each sensor must be known with sufficient exactness. In a quickly changing dynamic environment, the positions of sensors are not fixed, but must be adjusted to maintain optimal coverage. Therefore, the sensors need to autonomously align themselves in real time. This can be achieved by adding defined markers with given geometrical patterns to the environment which can be used for calibration and localization of each sensor. As soon as two sensors detect the same markers, their relative position to each other can be calculated. Additional anchor markers at fixed positions serve as global reference points for the base coordinate system.

In this paper we present a prototype for a self-aligning monitoring system based on a robot operating system (ROS) and Microsoft Kinect. This system is capable of autonomous real-time calibration relative to and with respect to a global coordinate system as well as to detect and track defined objects within the working area.
\end{abstract}

\section{Introduction}

The ability to autonomously acquire new knowledge through interaction with the environment has been in the focus of significant research in the field of dynamic work area. Challenging research topics arise in pose estimation, sensor alignment and object recognition. In order to accurately manipulate the objects in a dynamic work area, a reliable and precise vision system is required in a robotic system to detect and track workpieces and to monitor the operation of the robots to accomplish manufacturing tasks such as assembly planning (Ewert et al., 2012). Such a vision system not only has to be aware of the presence and location information in the working site, but also needs to have the information of its own real-time position and orientation as sensors.
Rather than being fixed, the vision system has to be able to move accordingly to provide a complete coverage in a dynamic scenario. To meet the above-mentioned requirements, we present a prototype for a self-aligning monitoring system based on an ROS and Microsoft Kinect. The main tasks of the vision system are autonomous self-calibration both relatively and with respect to the global coordinate system and target detection and tracking within the working area.

The proposed 3-D monitoring system, comprised of multiple Microsoft Kinects, is capable of self-alignment through calibrating Kinect both individually and as a stereo camera with reference to markers to obtain the relative location information between each other, as well as their pose in the global coordinate system. Two Kinects placed with a certain angle and distance with regards to each other can enable a 


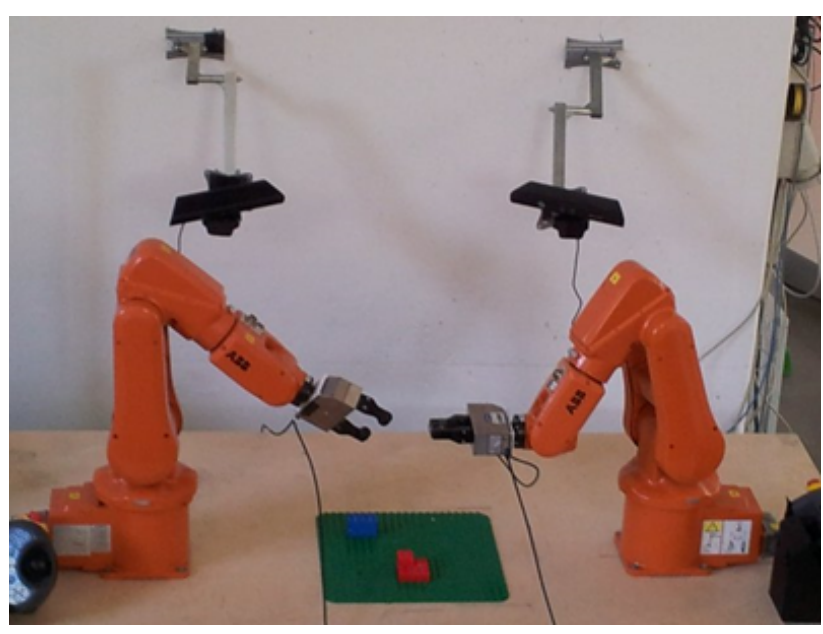

Figure 1. The test platform of the monitoring system.

full view of the working site if their image data are correctly aligned and fused. Experimental studies are carried out in the test platform which uses two Kinects and two ABB robots to represent the general case of multiple sensors and robots as Fig. 1 shows. While single 3-D sensors already provide detailed information within their field of view, complete coverage of a complete work area can only be achieved by relying on a multitude of these sensors. However, to provide useful information all data of each sensor must be aligned to each other, integrated and fused into an overall world picture. Therefore, it is of vital importance for sensors to be aware of not only its real-time pose in the real world but also their relative position and orientation to each other, so as to reconstruct a 3-D view of the working site.

To be able to align the data correctly, the position and orientation of each sensor must be known with sufficient exactness. To address this problem, a fixed marker is introduced into the system as an anchor. With the marker in sight, the Kinect matches the marker's location in the 2-D image with that in the real-world coordinate system to get the transformation from real-world coordinate system to the camera system. As individual Kinects are not fixed in the dynamic work area, there are circumstances where these Kinects do not detect the same geometrical marker for direct estimation of relative pose between each other or where one or both Kinects do not detect the anchor marker for self-positioning in the real world. Different relationships between Kinects and markers are considered and classified and corresponding solutions are presented in the following section.

Other than being able to be aware of its sensing element's pose relative to each other and with regards to the world coordinates, a vision based monitoring system is required to interpret a scene, which is defined as an instance of the real world consisting of one or more 3-D objects, to a determination of which 3-D objects are where in the scene. Therefore two main problems are involved: the first is object recognition, in which a label must be assigned to an object in the scene, indicating the category to which it belongs. The second involves the position and orientation estimation of the recognized object with respect to some global coordinate system attached to the scene. We adopt the viewpoint feature histogram (VFH) method to deal with the object recognition and six-degrees-of-freedom (6DoF) pose estimation will be discussed. It uses a two-dimensional Cartesian histogram grid as a world model, which is updated continuously and in real time with range data sampled by Kinect thus enabling realtime performance of the vision system.

The remainder of the paper is organized as follows: Sect. 2 presents a brief review of recent literature on object recognition approaches in industrial vision that are relevant to our proposed vision system. The architecture and workflow of industrial vision monitoring systems are discussed in Sect. 3. Software and hardware tools, sensor alignment and object recognition approaches that are used in assisting the development of the proposed vision systems are presented in Sect. 4. Section 5 summarizes the contribution of this work and plans for future work.

\section{Related work}

Much research attention has been drawn to workpiece position and orientation estimation in the industrial robot area, which is the primary requirement of industrial robot monitoring. A good variety of approaches have been proposed to solve object pose detection and their categorization. Literature differentiates between model- and view-based approaches (Bennamoun and Mamic, 2002; Bicego et al., 2005), feature- and appearance-based approaches or introduces several classes (Belongie et al., 2002). Among all other methods, the model of the object and the image data are represented by local geometric features. Geometric feature matching is used to interpret images through matching the model of object-to-data feature and estimating 3-D pose of the model. The shape, texture or the appearance of the object is always the center of attention. Because the object identification depends on this information to make reliable judgments by matching the model and scene data. We apply the model-based pose estimation approach in our research, which is done by matching geometric representations of a model of the object to those of the image data.

Besides object pose estimation, sensor self-positioning is another topic that researchers have been interested in and many efforts have been made in using and comparing marker and markerless pose estimation. Quite a few vision-based applications: camera calibration, augmented reality, etc., have benefited from the use of artificial 2-D and 3-D markers. These markers are designed to be easily detected and require very simple image processing operations. As to geometry, some applications are specially designed to avoid the trouble of estimating object pose. Typically, markerless object 


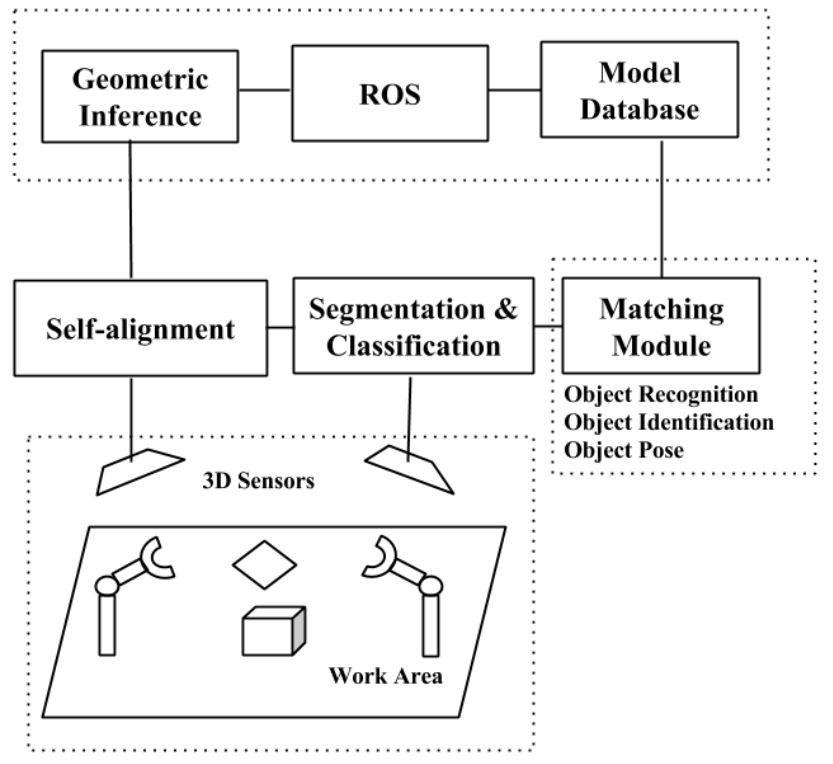

Figure 2. System architecture.

detection and pose estimation start with feature extraction (Canny, 1986; Forstner, 1994; Harris and Stephens, 1988; Smith and Brady, 1997). Other methods based on affine invariant regions determined around feature points were proposed (Kadir et al., 2004; Matas et al., 2002; Mikolajczyk and Schmid, 2004; Tuytelaars and Gool, 2004) in order to obtain invariance to out-of-plane rotations and translations. However, these algorithms are too time-consuming to meet the requirement of real-time computing speed. A registration method was proposed by State et al., 1996, using stereo images and a magnetic tracker. Vision techniques, multiple fiducial markers and square markers were used respectively for identifying 2-D matrix, markers robust tracking and fast tracking (Neumann et al., 1999; Rekimoto, 1998; Klinker et al., 1999). In our research, markers with distinct and simple geometrical patterns are used to attach on objects for recognizing and tracking, as they are easy to detect and recognize, thus achieving both robust and fast tracking.

We are proposing a real-time self-aligning multi-sensor vision monitoring system for a dynamic work area. Modelbased pose estimation approach and VFH method are applied for object recognition and $6 \mathrm{DoF}$ estimation; anchor markers are used for sensor self-alignment and simple geometrical markers are attached on objects to distinguish and track them, which enables the monitoring system to be aware of the real-time position and pose status of sensing elements, robots and objects in it.

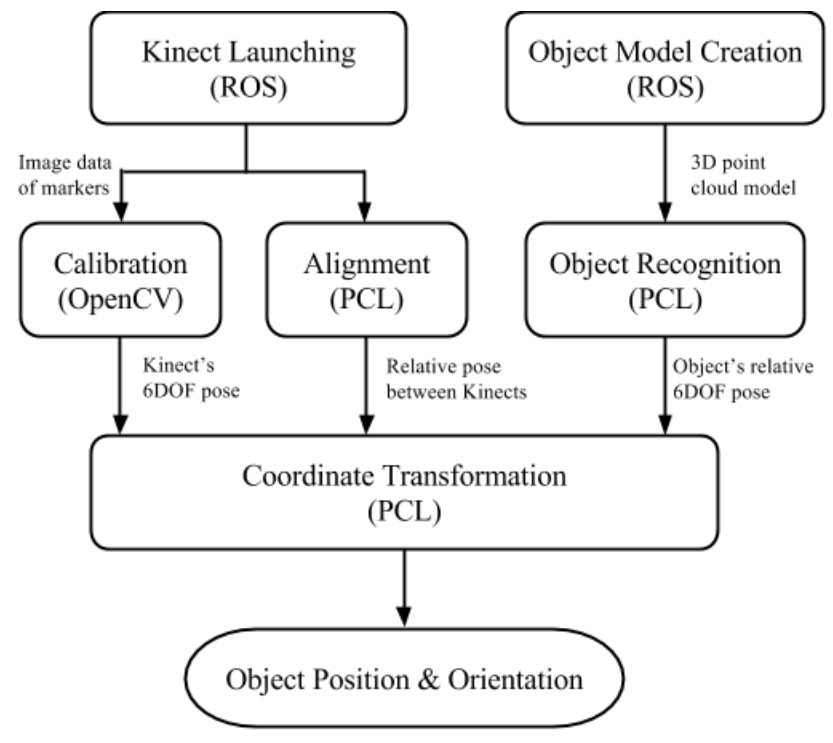

Figure 3. Workflow of the vision monitoring system.

\section{System overview}

\subsection{System architecture}

The monitoring mechanism of the proposed test system is shown in Fig. 2. The markers form geometric inference, which is used on a robot software development platform, ROS, to implement self-alignment of multi-sensors. An ROS is also used to create 3-D object point cloud models which compose a model database for object recognition and pose estimation by matching module after the overall scene image is processed by segmentation and classification module.

\subsection{Workflow}

Figure 3 is the workflow of monitoring object's movement in the work area.

- Kinect launching: An ROS camera driver launches Kinect and outputs 2-D/3-D image data.

- Calibration: calibrate a single Kinect with an anchor marker in work area. From the calibration the location of the points on the marker and its counterparts in the image, the transformation between marker and camera coordinates can be obtained. The location in the world coordinate is already known, thus, Kinect implements self-positioning.

- Alignment: align every Kinect pair as a stereo camera. As two Kinects detect the same marker, they register their captured images at the corresponding points and compute the relative position and orientation between the Kinects, thus align the image from the two Kinects to visualize the work area. 
- Object model creation: create 3-D point-cloud model of object for later recognition and alignment.

- Object recognition: recognize and position the object from the scene. Object relative position and orientation will be obtained through aligning the object model to the point cloud of current scene.

- Coordinate transformation: transform object pose which is relative to scene in camera coordinate system to global coordinate system.

\section{Tools and methods}

\subsection{Tools}

\subsubsection{Kinect}

The robot has to rely on its sensory feedback to build a model of its surroundings. The 3-D sensor used in our research is Microsoft Kinect. It is able to capture the surrounding world in 3-D by combining the information from depth sensors and a standard RGB camera as shown in Fig. 4. The result of this combination is an RGB-D image with $640 \times 480$ resolution, where each pixel is assigned color information and depth information. In ideal conditions the resolution of the depth information can be as high as $3 \mathrm{~mm}$, using 11 bit resolution. Kinect works with $30 \mathrm{~Hz}$ frequency for both RGB and depth cameras. On the left side of the Kinect is a laser infrared light source that generates electromagnetic waves with the wavelength of $830 \mathrm{~nm}$. Information is encoded in light patterns that are deformed as the light reflects from objects in front of the Kinect. Based on these deformations captured by the sensor on the right side of RGB camera, a depth map is created. According to the light coding technology PrimeSense, this is not the time-of-flight method used in other 3-D cameras (Tolgyessy and Hubinsky, 2011). The interaction space is defined by the field of view of the Kinect cameras. To increase the possible interaction space, the built-in tilt motor supports an additional +27 and $-27^{\circ}$, which also allows for the dynamic interaction in front of the sensor.

\subsubsection{ROS}

Robot operating system (ROS) (http://www.ros.org) is a software framework for robot software development, providing standard operating system services such as hardware abstraction, low-level device control implementation of commonly used functionality, message passing between processes, and package management. It is based on a graph architecture where nodes that receive, post and process messages from sensors, control, state, planning and actuactor.

An ROS is composed of two main parts: the operating system ROS as described above and ROS-pkg, a suite of user contributed packages that implement functionality such as simultaneous localization and mapping, planning, perception,

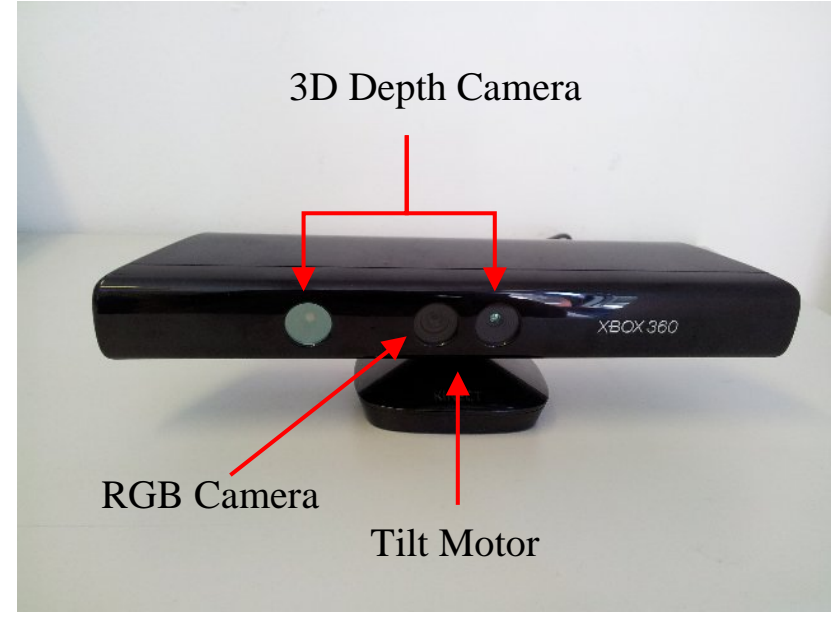

Figure 4. Kinect.

simulation, etc. The Kinect node package provides a driver for using the Kinect RGB-D sensor with an ROS, which launches an OpenNI device and loads all nodelets to convert raw depth/RGB/IR streams to depth image, disparity image and registered point clouds. So it outputs point clouds, RGB image messages and its associated camera information for calibration, object recognition and alignment.

\subsubsection{PCL}

The point cloud library (PCL) (http://pointclouds.org) is a large-scale, open project for 2-D/3-D image and point cloud processing. The PCL framework contains numerous state-ofthe-art algorithms including filtering, feature estimation, surface reconstruction, registration, model fitting and segmentation. These algorithms can be used, for example, to filter outliers from noisy data, stitch 3-D point clouds together, segment relevant parts of a scene, extract key points and compute descriptors to recognize objects in the world based on their geometric appearance, and create surfaces from point clouds and visualize.

\subsubsection{OpenCV}

OpenCV (Open Source Computer Vision Library) (OpenCV.org) is an open source computer vision and machine learning software library. OpenCV was built to provide a common infrastructure for computer vision applications and to accelerate the use of machine perception in the commercial products. The library has a comprehensive set of both classic and state-of-the-art computer vision and machine learning algorithms. 


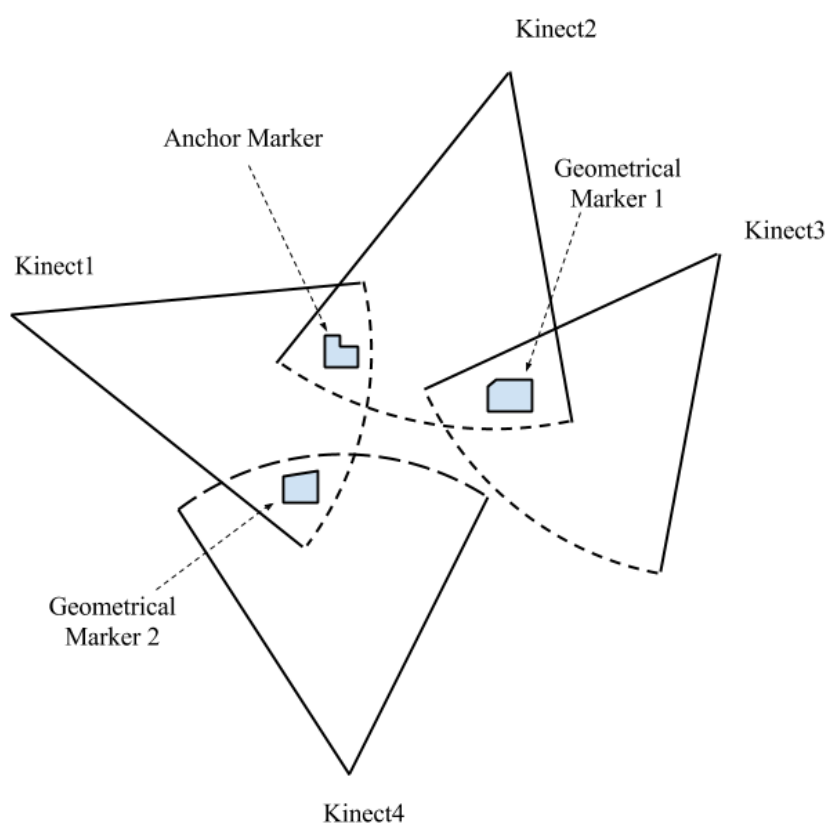

Figure 5. Four possible relative cases of multiple cameras.

\subsection{Methods and mechanism}

\subsubsection{Sensor alignment}

In the proposed vision monitoring system, multiple sensors are used and must be aligned to each other and fused into an overall world picture. In order to align the sensing data accurately, the position and orientation of each sensor are of priority for aligning the sensing data accurately. Our work employs two types of 2-D markers respectively fixed on the work area as landmarks for camera self-positioning and attached on the objects as name tags for object identification, namely anchor markers and geometrical markers. The introduction of anchor markers and geometrical markers ensures the reconstruction of the whole scene of the work area. Instead of being fixed in the work area, Kinect moves up and down, left and right on its base to obtain visual information of the work scene from different viewpoints. Therefore, the spatial relationships of anchor markers, geometrical markers and Kinects vary from time to time. The relative pose of Kinect can be generally summarized and classified into four cases, as shown in Fig. 5:

1. Kinect 1 and Kinect 2 have at least one anchor marker in their intersected vision area.

2. Kinect 2 and Kinect 3 have at least one distinguishing marker and no anchor marker in their intersected vision area.

3. Two Kinects have no common marker in their intersected vision area:

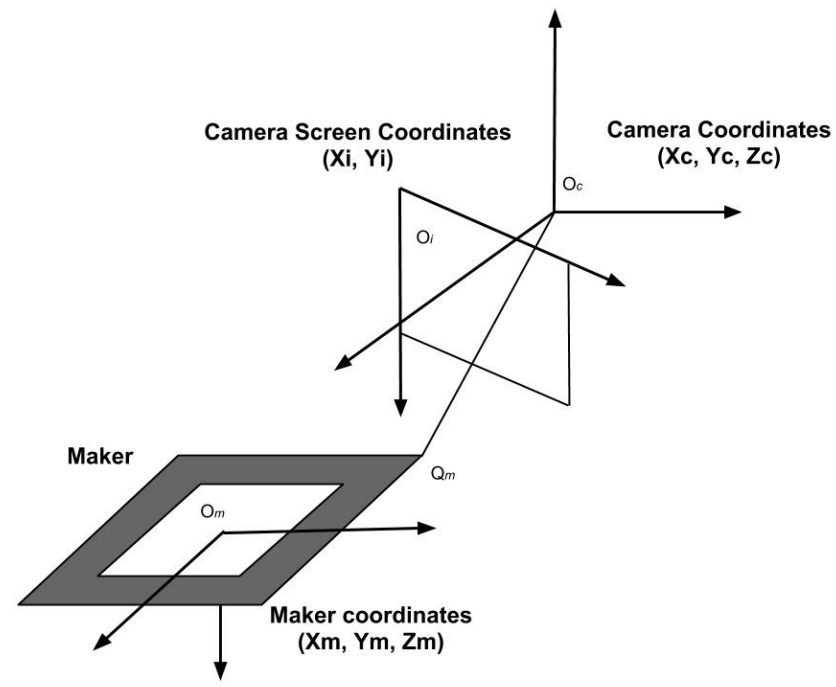

Figure 6. The relationship between marker and camera coordinates.

a. Kinect 1 can position itself by an anchor marker; Kinect 3 has no anchor marker in its sight but a geometrical marker.

b. Both Kinect 3 and 4 detect no anchor marker but geometrical markers.

For case 1, Kinect 1 and 2 can use the anchor marker in sight for their own 3-D pose estimation by relating camera measurements with measurements in the real, three-dimensional world. In this model, a marker scene view is formed by projecting 3-D points of the marker into the image plane using a perspective transformation as Fig. 6 shows.

Projective transform maps the points $Q_{m}$ in the global world coordinate system $\left(X_{m}, Y_{m}, Z_{m}\right)$ to the points on the image plane with coordinates $\left(x_{i}, y_{i}\right)$ and to the points on camera plane with coordinates $\left(X_{c}, Y_{c}, Z_{c}\right)$. The projection from global world coordinate system to camera image coordinate system can be summarized as in Eq. (1):

$$
\left[\begin{array}{c}
X_{c} \\
Y_{c} \\
Z_{c} \\
1
\end{array}\right]=\left[\begin{array}{cccc}
r_{11} & r_{12} & r_{13} & t_{1} \\
r_{21} & r_{22} & r_{23} & t_{2} \\
r_{31} & r_{32} & r_{33} & t_{3} \\
0 & 0 & 0 & 1
\end{array}\right]\left[\begin{array}{c}
X_{m} \\
Y_{m} \\
Z_{m} \\
1
\end{array}\right] .
$$

For case 2, Kinect 2 and 3 capture the same non-anchor marker. For any given 3-D point $P$ in object coordinates, we can put $P$ in the camera coordinates $P_{1}=\mathbf{R}_{1} P+\boldsymbol{T}_{1}$ and $P_{\mathrm{r}}=\mathbf{R}_{\mathrm{r}} P+\boldsymbol{T}_{\mathrm{r}}$ for the left and right cameras, respectively. It is also evident that the two views of $P$ (from the two cameras) are related to $P_{1}=\mathbf{R}^{T}\left(P_{\mathrm{r}}-\boldsymbol{T}\right)$, where $\mathbf{R}$ and $\boldsymbol{T}$ are, respectively, the rotation matrix and translation vector between the cameras. Taking these three equations and solving for the rotation and translation separately yields the following 


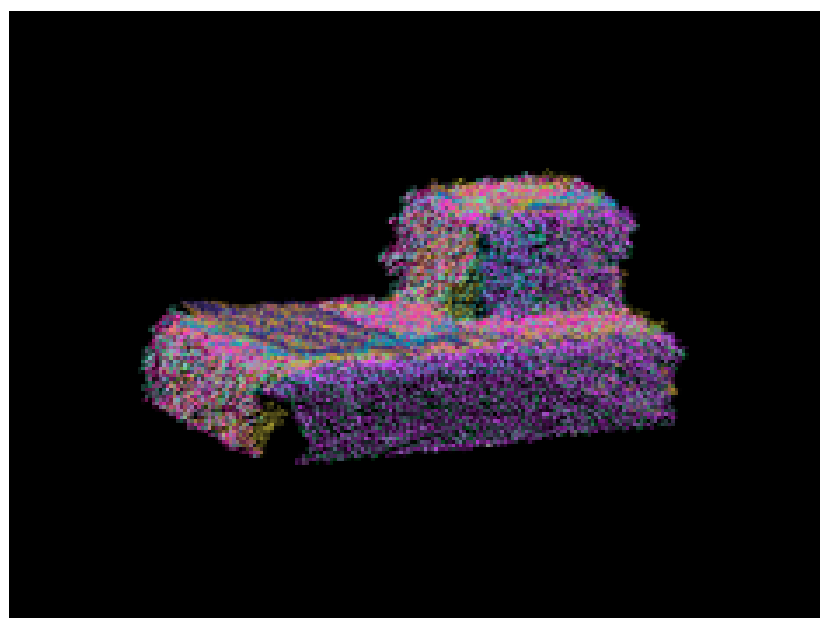

Figure 7. Overlapping of 7 point clouds at different viewpoints.

simple relations (OpenCV.org):

$$
\begin{aligned}
& \mathbf{R}=\mathbf{R}_{\mathrm{r}}\left(\mathbf{R}_{1}\right)^{T}, \\
& \boldsymbol{T}=\boldsymbol{T}_{\mathrm{r}}-\mathbf{R} \boldsymbol{T}_{1} .
\end{aligned}
$$

Then the relative rotation and translation from Kinect 1 to Kinect 2 can be obtained, and in the chain of Kinects that detect the same marker with Kinect 2 directly or indirectly, there must be one that has an anchor marker in vision range. Therefore, the second case can be solved in the same way as case 1, only with the corresponding transformations.

For case 3, Kinect 1 and Kinect 3 do not have the same marker in their vision ranges. We apply a similar strategy here by searching for an anchor marker in the chain composed of overlapped Kinects to estimate the $6 \mathrm{DoF}$ pose of at least one Kinect and then to make pose estimation of others through coordinate transformation.

\subsubsection{Object Recognition and 6DoF pose estimation}

Object recognition is the process of automatic identification and localization of objects from the sensed images of scenes in the real world. For object recognition in this system, scene point clouds with the object's presence are downsampled by corresponding sampling algorithm from PCL for analysis and computation. To obtain the surface normals of the specified input point clouds, Kd-Tree (http://ros.org) is used to search for neighboring point and the radius that defines each point's neighborhood. The VFH (http://pointclouds.org) descriptor is employed as a representation for point cluster recognition and its $6 \mathrm{DoF}$ pose estimation. The computation of VFH descriptors is implemented from the input point cloud and its surface normals. The resulted features are invariant to image scaling, translation, rotation and partially invariant to illumination changes and affine or 3-D projection. With the normals and local feature descriptors, the object point cloud model is aligned into the current scene cloud

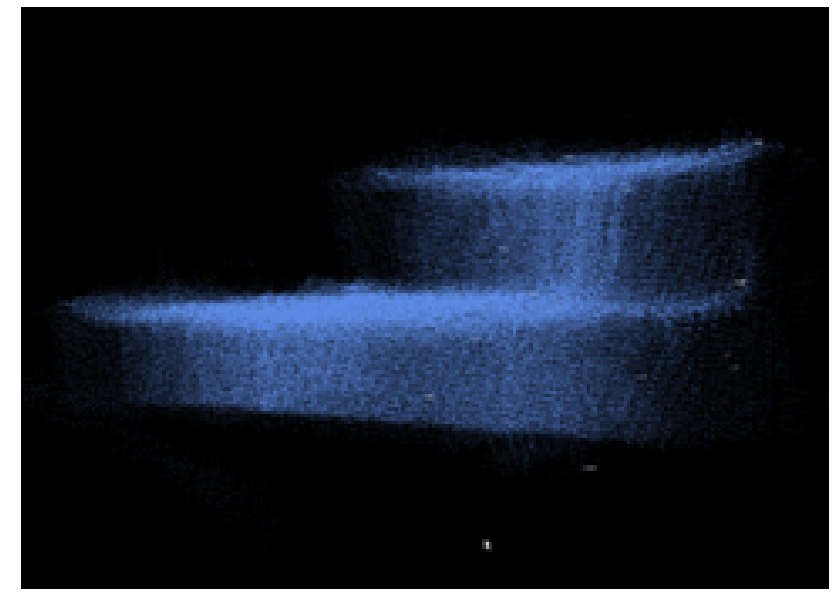

Figure 8. Merging of 34 point clouds at 34 different viewpoints.

to get final transformation and a fitness score to evaluate the aligning results.

Object recognition is achieved by matching features derived from the scene with stored object model representations. One of the most common ways to create the object model for recognition is to extract the target as a cluster from the point cloud. However, in this way only a partial model is created out of the object, which provides very limited information for object identification.

\section{Model creation}

In this research, the approach adopted to create a 3-D point cloud model from an object is to use an object recording API of the package RoboEarth from an ROS along with a Kinect camera and a marker pattern. The target object is placed in the middle of the marker template and either the camera or marker pattern and object are moved to record a complete pose. It is always a better idea to move the object, otherwise the illumination might not be constant and therefore color effects might arise.

Figure 7 shows the overlapping of point clouds of the object captured at seven different viewpoints and all the point clouds are created at 34 different viewpoints and are finally processed and merged into one 3-D point cloud model as Fig. 8 shows.

\section{Normal estimation}

Given a geometric surface, it is usually trivial to infer the direction of the normal at a certain point on the surface as the vector perpendicular to the surface in that point. The problem of determining the normal to a point on the surface is approximated by the problem of estimating the normal of a plane tangent to the surface, which in turn becomes a least-square plane fitting estimation problem. The solution for estimating 
the surface normal is therefore reduced to an analysis of the eigenvectors and eigenvalues of a covariance matrix created from the nearest neighbors of the query point. More specifically, for each point $p_{i}$, we assemble the covariance matrix $\mathbf{C}$ as follows:

$\mathbf{C}=\frac{1}{k} \sum_{i=1}^{k}\left(p_{i}-\bar{p}\right) \cdot\left(p_{i}-\bar{p}\right)^{T}$,

$\mathbf{C} \cdot \bar{v}_{j}=\lambda_{j} \cdot \bar{v}_{j}, j \in\{0,1,2\}$,

where $k$ is the number of point neighbors considered in the neighborhood of $p_{j}, p$ represents the 3-D centroid of the nearest neighbors, $\lambda_{j}$ is the $j$ th eigenvalue of the covariance matrix, and $v_{j}$ the $j$ th eigenvector (Bradski and Kaehler, 2008).

\section{Feature description}

Features define individual components of an image and can be categorized into two major groups: global features and local features. Global features are defined as properties of an image based on the whole image. Local features are defined as properties of an image based on a component of the image and these will be used for object recognition. Therefore, we need a way to describe the features of an image.

VFH descriptor is a novel representation for point cluster recognition and its $6 \mathrm{DoF}$ pose estimation. VFH has its roots in FPFH (Fast Point Feature Histograms) descriptor and add in viewpoint variance while retaining invariance to scale. The main idea of object recognition through VFH descriptors is to formulate the recognition problem as a nearest neighborhood estimation problem. Let $p_{\mathrm{c}}$ and $n_{\mathrm{c}}$ be the centroids of all surface points and their normals of a given object partial view in the camera coordinate system (with $\left.\left\|n_{\mathrm{c}}\right\|=1\right)$. Then $\left(u_{i}, v_{j}, w_{i}\right)$ defines a Darboux coordinate frame for each point $p_{i}$ :

$u_{i}=n_{\mathrm{c}}$,

$v_{i}=\frac{p_{i}-p_{\mathrm{c}}}{\left\|p_{i}-p_{\mathrm{c}}\right\|} \times u_{i}$

$w_{i}=u_{i} \times v_{i}$.

The normal angular deviations $\cos \left(\alpha_{i}\right), \cos \left(\beta_{i}\right)$ and $\cos \left(\varphi_{i}\right)$ for each point $p_{i}$ and its normal $n_{i}$ given by

$\cos \left(\alpha_{i}\right)=v_{i} \cdot n_{i}$

$\cos \left(\beta_{i}\right)=n_{i} \cdot \frac{p_{\mathrm{c}}}{\left\|p_{\mathrm{c}}\right\|}$,

$\cos \left(\varphi_{i}\right)=u_{i} \cdot \frac{p_{i}-p_{\mathrm{c}}}{\left\|p_{i}-p_{\mathrm{c}}\right\|}$

$\theta_{i}=a \tan 2\left(w_{i} \cdot n_{i}, u_{i} \cdot n_{i}\right)$.

Note that $\cos \left(\alpha_{i}\right), \cos \left(\beta_{i}\right)$ and $\theta_{i}$ are invariant to viewpoint changes, given that the set of visible points does not change.
For $\cos \left(\alpha_{i}\right), \cos \left(\beta_{i}\right)$ and $\theta_{i}$ histograms with 45 bins each are computed and a histogram of 128 bins for $\cos \left(\beta_{i}\right)$, thus the VFH descriptor has 263 dimensions (Aldoma and Vincze, 2011).

\section{Pose estimation}

As the point cloud data of an object model is stored and the corresponding Kd-tree representation is built up, objects are extracted from the given scene as clusters and for each of them, an individual cluster; for each cluster, their VFH descriptor from the current camera position is computed for searching for candidates in the trained Kd-tree. After find the best candidate for recognition, the position and orientation of the object that the model represents can be determined by registering the model to the scene point cloud.

\section{Conclusions}

In this paper, we have introduced a new approach for work area monitoring in a dynamic environment using multiple 3D self-aligning Kinects. The anchor marker is used to calibrate Kinect to correct for the main deviations from the pinhole model that Kinect uses, to obtain the transformations from a global coordinate system to a camera coordinate system and relative position and orientation between the Kinects. In this way, Kinect is able to have an awareness of its own positions and 6DoF poses as well as the object's location in the working scenario at any moment, enabling robots to accommodate changes in the workpiece position/orientation and to perform complex operations like automated assembling and sorting. Simple geometrical markers are used to distinguish objects, which achieves robust and fast tracking of objects in dynamic work sites. In conclusion, addressing the requirements of real-time monitoring of a dynamic industrial production area, the proposed vision monitoring system is able to provide overall vision of the work area and estimate 6DoF pose of multiple objects with defined geometrical markers and anchor markers.

To evaluate and optimize the performance of our proposed approaches in this vision system, we will involve the following aspects as future research topics. Firstly, adopt color information for object recognition and extraction; secondly, implement boundary analysis using the combination of a photogrammetric processing algorithm and point cloud spatial information; thirdly, compare the results of using different models to align to scene image: 3-D CAD model, model generated based on both digital image and point cloud obtained by depth camera, scanned object 3-D point cloud model and object model extracted from the scene image.

Edited by: R. Tutsch

Reviewed by: two anonymous referees 


\section{References}

Aldoma, A. and Vincze, M.: CAD-Model Recognition and 6DOF Pose Estimation Using 3D Cues, IEEE International Conference on Computer Vision Workshops, 585-592, 2011.

Belongie, S., Malik, J., and Puzicha, J.: Shape matching and object recognition using shape contexts, IEEE T. Pattern Anal., 24, 509522, 2002.

Bennamoun, M. and Mamic, G. J.: Object recognition: fundamentals and case studies, London, Springer, 2002.

Bicego, M., Castellani, U., and Murino, V.: A hidden Markov model approach for appearance-based 3D object recognition, Pattern Recognition, 26, 2588-2599, 2005.

Bradski, G. and Kaehler, A.: Learning OpenCV: Computer Vision with the OpenCV Library, O'Reilly, 378-386, 2008.

Canny, J. F.: A computational approach to edge detection, IEEE T. Pattern Anal., 8, 679-698, 1986.

Ewert, D., Mayer, M., Schilberg, D., and Jeschke, S.: Adaptive assembly planning for a nondeterministic domain, Conference Proceedings of the 4th International Conference on Applied Human Factors and Ergonomics, 1253-1262, 2012.

Forstner, W.: A framework for low-level feature extraction, European Conf. on Computer Vision, 383-394, 1994.

Harris, C. and Stephens, M.: A combined corner and edge detector, Proc. of the 4th Alvey Vision Conf., 147-151, 1988.

Kadir, T., Zisserman, A., and Brady, M.: An affine invariant salient region detector, European Conf. on Computer Vision, 2004.

Klinker, G., Stricker, D., and Reiners, D., Augmented Reality: A Balancing Act between High Quality and Real-Time Constraints, Proceedings of ISMR '99, 325-346, 1999.
Matas, J., Chum, O., Urban, M., and Pajdla, T.: Robust wide baseline stereo from maximally stable extremal regions, British Machine Vision Conf., 2002.

Mikolajczyk, K. and Schmid, C.: Scale \& affine invariant interest point detectors, Int. J. Comput. Vision, 60, 63-86, 2004.

Neumann, U., You, S., Cho, Y., and Lee, J.: Augmented Reality Tracking in Natural Environments, Mixed Reality - Merging Real and Virtual Worlds, Ohmsha and Springer-Verlag, 101-130, 1999.

OpenCV.org: http://docs.opencv.org/modules/calib3d/doc/camera_ calibration_and_3d_reconstruction.html (last access: 9 April 2013).

PCL: http://www.pointclouds.org/documentation/tutorials/ (last access: 9 April 2013), http://ros.org, http://www.ros.org/wiki/ (last access: 9 April 2013).

Rekimoto, J.: Matrix: A Realtime Object Identification and Registration Method for Augmented Reality, Proceedings of Asia Pacific Computer Human Interaction, 15-17, 1998.

Smith, S. M. and Brady, J. M.: A New Approach to Low Level Image Processing, Int. J. Comput. Vision, 23, 45-78, 1997.

State, A., Hirota, G., Chen, D. T., Garrett, W. F., and Livingston, M. A.: Superior Augmented Reality Registration by Integrating Landmark Tracking and magnetic Tracking, Proceedings of SIGGRAPH96, 429-446, 1996.

Tolgyessy, M. and Hubinsky, P.: The Kinect Sensor in Robotics Education, In Proceedings of 2nd International Conference on Robotics in Education. Vienna, Austria, 143-146, 2011.

Tuytelaars, T. and Gool, L. Vn.: Matching Widely Separated Views Based on Affine Invariant Regions, Kluwer Academic Publishers, 2004. 